\title{
An investigation into the effect of fabrication parameter variation on the characteristics of screen-printed thick-film silver/silver chloride reference electrodes
}

\author{
F.K. Atkinson, M. Glanc, P. Boltryk and M. Sophocleous \\ School of Engineering Sciences, University of Southampton, Southampton, UK, and \\ E. Garcia-Breijo \\ Instituto de Reconocimiento Molecular y Desarrollo Tecnológico, Universidad Politécnica de Valencia, Valencia, Spain
}

\begin{abstract}
Purpose - The purpose of this paper is to show how the fabrication parameters of screen-printed thick-film reference electrodes have been experimentally varied and their effect on device characteristics investigated.

Design/methodology/approach - The tested devices were fabricated as screen-printed planar structures consisting of a silver back contact, a silver/ silver chloride interfacial layer and a final salt reservoir layer containing potassium chloride. The fabrication parameters varied included deposition method and thickness, salt concentration and binder type used for the final salt reservoir layer. Characterisation was achieved by monitoring the electrode potentials as a function of time following initial immersion in test fluids in order to ascertain initial hydration times, subsequent electrode drift rates and useful lifetime of the electrodes. Additionally, the effect of fabrication parameter variation on electrode stability and their response time in various test media was also investigated.

Findings - Results indicate that, although a trade-off exists between hydration times and drift rate that is dependent on device thickness, the initial salt concentration levels and binder type also have a significant bearing on the practical useful lifetime. Generally speaking, thicker devices take longer to hydrate but have longer useful lifetimes in a given range of chloride environments. However, the electrode stability and response time is also influenced by the type of binder material employed for the final salt reservoir layer.

Originality/value - The reported results help to explain better the behaviour of thick-film reference electrodes and contribute towards the optimisation of their design and fabrication for use in solid-state chemical sensors.
\end{abstract}

Keywords Electrodes, Films (states of matter), Silver, Chloride, Salt, Electrochemistry

Paper type Research paper

\section{Introduction}

The usage of screen-printed (thick-film) fabrication methods for chemical and environmental sensor arrays has been growing steadily in recent years with a wide range of devices being reported. Although the use of a conventional electrolyte filled commercially obtained reference electrode is often employed in the characterisation of these devices, some have employed a screen-printed reference electrode, generally based on a silver/ silver halide electrode. Sometimes, the thick-film reference electrode takes the form of a pseudo-reference (Boujtita et al., 2000; Andreescu et al., 2002; Matsumoto et al., 2002) whereby the silver/silver halide electrode surface is in direct contact with the analyte. As such the reference electrode derives its stability from knowledge of the environmental matrix into which it is deployed such that the stability of the electro-potential of the

The current issue and full text archive of this journal is available at www.emeraldinsight.com/1356-5362.htm

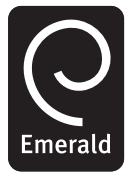

Microelectronics International

28/2 (2011) 49-52

Emerald (C) Emerald Group Publishing Limited [ISSN 1356-5362]

[DOI 10.1108/13565361111127368] reference electrode is unimportant. In other instances, the construction of the reference electrode attempts to mimic the operation of a conventional electrolyte-filled reference electrode usually through the immobilising of a salt reservoir on top of the reference electrode material (Mroz et al., 1998; Tymeckia et al., 2004; Maminska et al., 2006).

The devices reported here are fabricated as screen-printed planar electrodes with a salt containing layer deposited on top of the electrode to give some measure of electro-potential stability in a similar manner to that of a conventional electrolyte filled reference electrode. Early attempts at fabricating electropotentially stable devices (Cranny and Atkinson, 1998) concentrated on the use of a polymer bound potassium chloride filled layer that was screen printed onto a silver/silver chloride layer. These devices have previously been employed successfully on chemical and environmental arrays for use in a range of application areas (Mihell and Atkinson, 1998; Atkinson et al., 1999; Gac et al., 2002, 2004; Glasspool and Atkinson, 2003). In all these cases, the analyte matrix was relatively well understood and allowance could be made for electrode potential drift. However, recent commercial interest in the use of thickfilm chemical sensor arrays in longer term applications in relatively unknown matrices requires that they have much better electro-potential stability than that previously attained. 


\section{Electrode fabrication}

The test electrodes employed for testing here were fabricated on $50.8 \mathrm{~mm}^{2}, 0.625 \mathrm{~mm}$ thick 96 percent alumina substrates (Coorstech). The substrates were prescribed with laser lines to produce substrates that could be used for the simultaneous fabrication of six electrodes at a time (Figure 1). Individual electrodes could then be snapped from the substrate along the laser scribe lines.

The cross-sectional view of the individual reference electrodes shown in Figure 2 reveals the construction as a succession of screen-printed layers commencing with a silver conductor layer (ESL 9912) onto which is deposited an insulating glass layer (ESL 4905) with a $5 \times 5 \mathrm{~mm}$ window that exposes the underlying silver layer. A layer of glass bound silver/ silver chloride paste (GEM C61003P7) is then printed and fired on top of the silver exposed in the window before a second layer of insulating glass with the same $5 \times 5 \mathrm{~mm}$ window opening as before is deposited on top. Finally, a layer containing potassium chloride is deposited onto the silver/silver chloride layer.

Two different types of potassium chloride layer were tested with one type consisting of a silicone-based polymer paste (ESL 242SB) containing different concentrations of potassium

Figure 1 Strips of thick-film reference electrodes fabricated on a common "snapstrate" showing silver electrical connections at bottom and window exposing silver/silver chloride electrode at top

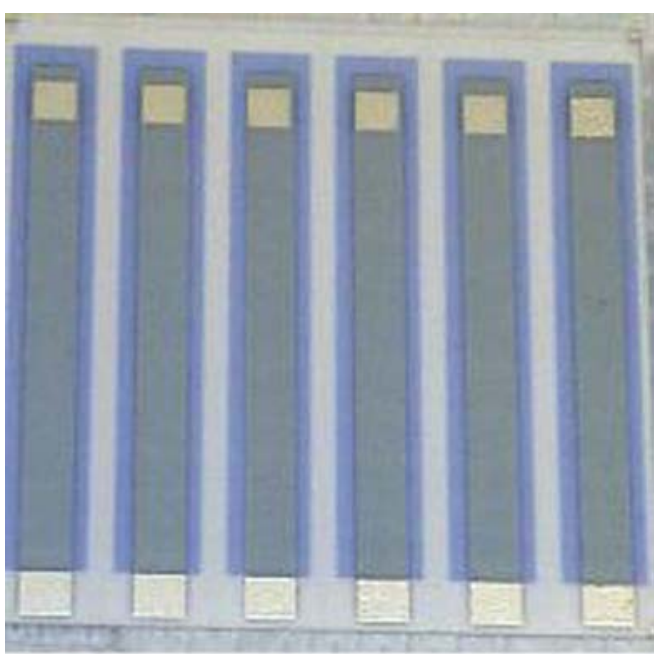

Figure 2 Thick-film reference electrode construction

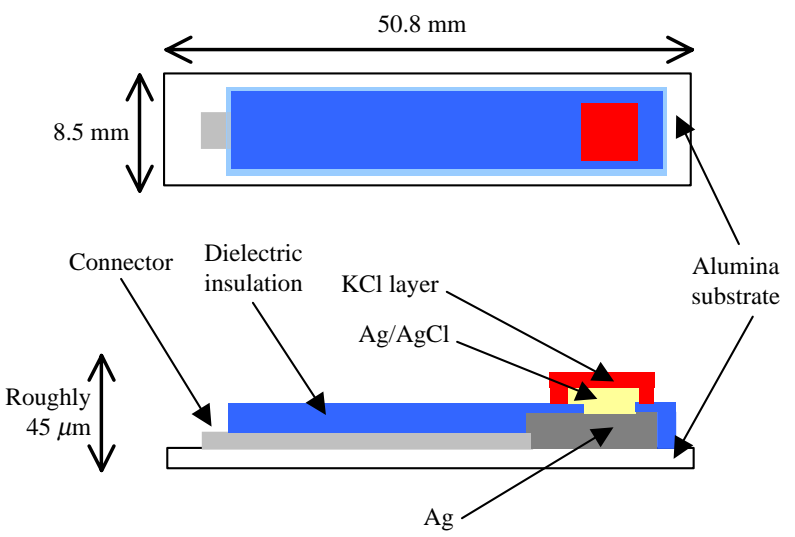

chloride of approximately 6 percent, 3 percent and 0.6 percent by weight and the other a solgel made from commercially obtained gelatine dissolved in different concentrations of potassium chloride solution in deionised water (saturated solution, and said saturated solution further diluted with deionised water in ratios of $1: 2$ and $1: 10$ ). The polymer bound salt reservoir was screen printed onto the silver/silver chloride electrodes while the solgel solutions were applied using a dropper to give an approximate droplet volume of $3 \mathrm{ml}$ per electrode.

\section{Electrode testing}

Electrode preparation

The electrodes were initially hydrated in a dibasic sodium phosphate/monobasic potassium phosphate $\mathrm{pH} 7$ buffer solution (Oakton) and their electrode potentials were measured with respect to a commercial double junction electrolyte filled reference electrode (Beckman Coulter A57193) using a Keithley 2000 Digital Multimeter. The electrodes were connected to the multimeter in batches of ten at a time using a K2000 scan card and held in solution using a mounting plate as shown in Figure 3.

\section{Test solutions}

Different concentrations of potassium chloride test solutions were made by first of all dissolving potassium chloride $(\mathrm{BDH}$ $101984 \mathrm{~L}$ AnalaR) in deionised water until saturation and then diluting this solution in the ratios of $1: 10,1: 100$ and 1:1000. These solutions were then designated SAT, SAT 1:10, SAT 1:100 and SAT 1:1000, respectively.

The electrodes to be tested were then immersed in each of the test solutions in turn and allowed to stabilise for approximately $1 \mathrm{~h}$ before their individual potentials versus the commercial reference electrode were recorded.

\section{Results}

The average electro-potentials of the thick-film reference electrodes (plotted versus the commercial reference electrode) hydrating in $\mathrm{pH} 7$ buffer as a function of time were as shown in Figure 4.

Figure 3 Testing of thick-film reference electrodes versus commercial reference electrodes

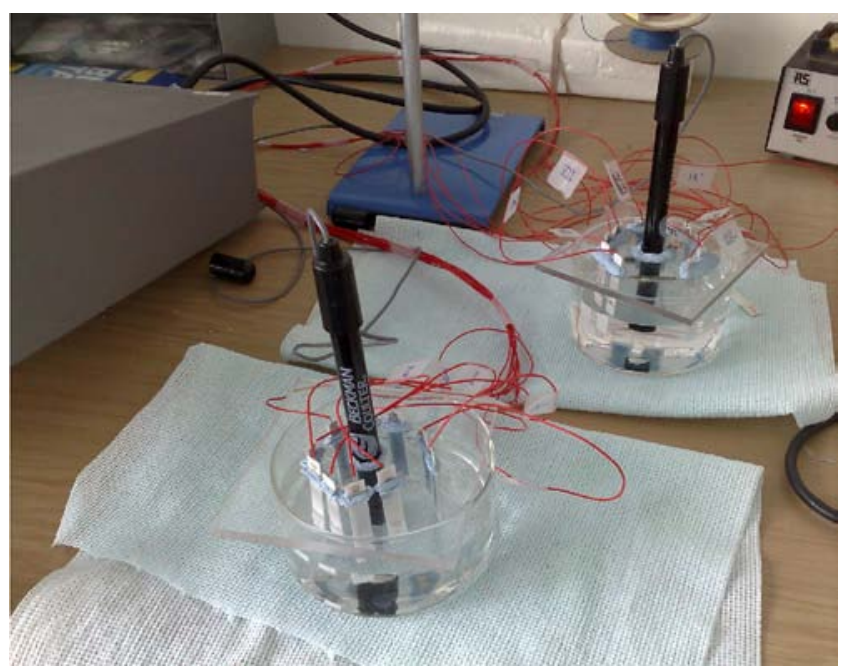


Figure $4 \mathrm{ESL} 242-\mathrm{SB} / \mathrm{KCl}$ electrodes initially hydrating and then drifting in $\mathrm{pH} 7$ buffer

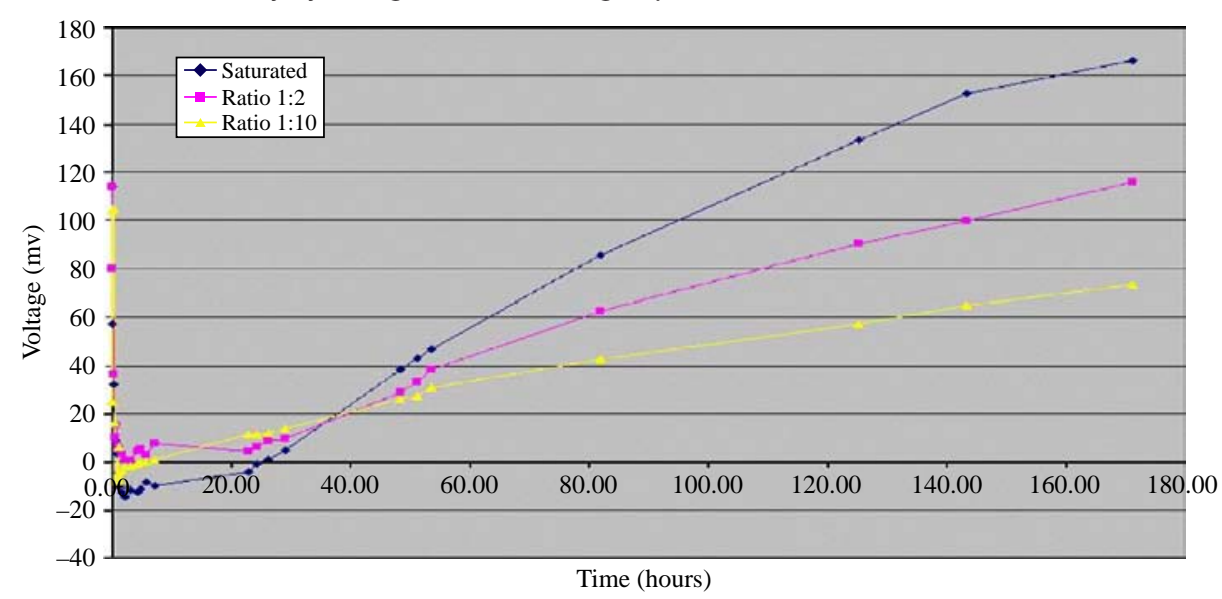

After an initial period of electro-potential instability lasting approximately $1 \mathrm{~h}$ the electrodes hydrate and remain relatively stable for a period of approximately $24 \mathrm{~h}$. After this initially stable period they begin to drift at different rates. It can be seen from Figure 4 that the higher the initial concentration of potassium chloride contained in the electrodes the faster they drift.

The average electro-potential response of the electrodes to successive immersions in test solutions of deionised water containing potassium chloride concentrations of A: SAT, B: SAT 1:10, C: SAT 1:100 and D: SAT 1:1000 are as shown in Figure 5.

The electrodes exhibited potential steps of approximately $10 \mathrm{mV}$ per decade of test solution potassium chloride concentration with the step sizes being noticeably smaller for the electrodes containing higher initial concentrations of potassium chloride. This should be compared with the step sizes for a bare silver/silver chloride electrode (as shown in Figure 6 for example) of typically between 50 and $100 \mathrm{mV}$ per decade of potassium chloride concentration. It should also be noted that the electrode potentials became more negative with decreasing potassium chloride solution concentration.

Figure 6 shows the averaged response of the hydrogel electrodes to successive immersions in solutions of deionised water containing the same potassium chloride concentrations as previously described and designated A-D.
The responses of the electrodes showed relatively large potential steps (between 50 and $100 \mathrm{mV}$ ) for each electrode type when moving from SAT to SAT 1:10 but much lower potential steps thereafter when moving between lower concentration solutions. A bare silver/silver chloride electrode is shown on the same graph for comparison and exhibits step sizes of approximately $100 \mathrm{mV}$ per decade change in potassium chloride solution concentration. It should also be noted that for the hydrogel electrodes the electrode potentials became more positive with decreasing potassium chloride solution concentration.

\section{Conclusions}

The reason that the ESL242SB/KCl electrodes gave a potential response to different concentrations of chloride solution that was the opposite of the both the bare $\mathrm{Ag} / \mathrm{AgCl}$ and the solgel electrodes is thought to be due to a combined response of the silicone polymer itself and the $\mathrm{KCl}$ salt concentration in the polymer.

The fact that higher concentrations of $\mathrm{KCl}$ gave lower step sizes would tend to suggest that increasing the $\mathrm{KCl}$ concentration to an optimum level will result in electrodes that have responses to varying chloride concentration that are almost flat, whereby the $\mathrm{KCl}$ response "cancels out" the silicone polymer response.

Figure $5 \mathrm{ESL} 242-\mathrm{SB} / \mathrm{KCl}$ electrode potentials in different concentration $\mathrm{KCl}$ solutions

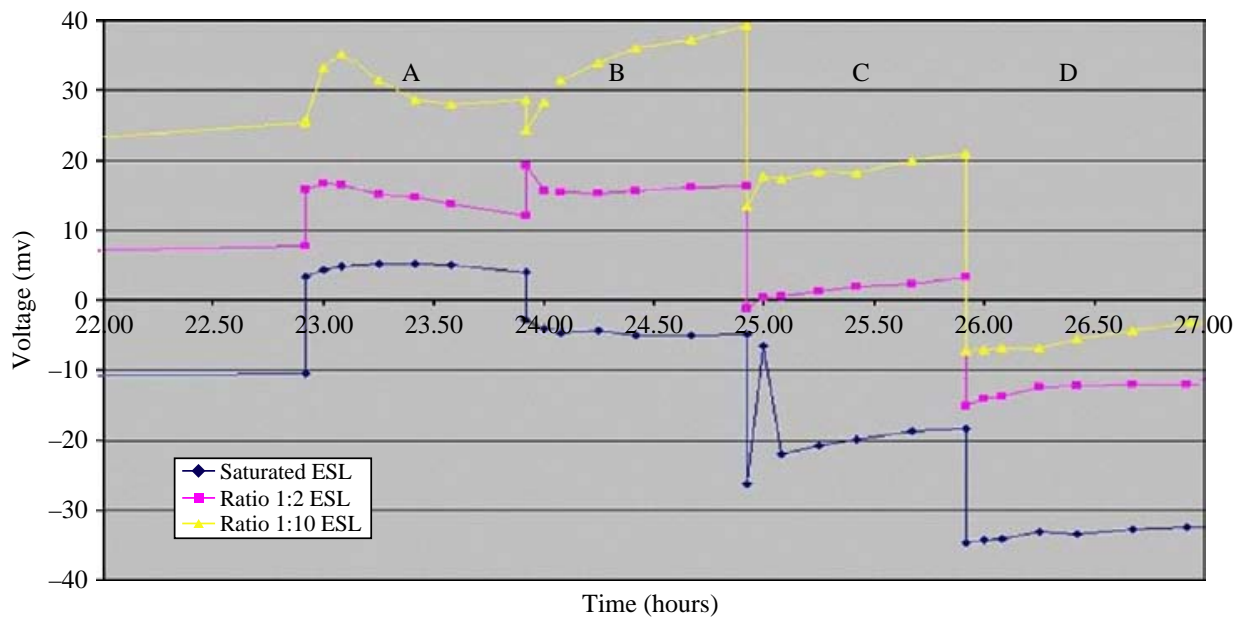


Figure 6 Hydrogel $/ \mathrm{KCl}$ electrode potentials in different concentration $\mathrm{KCl}$ solutions

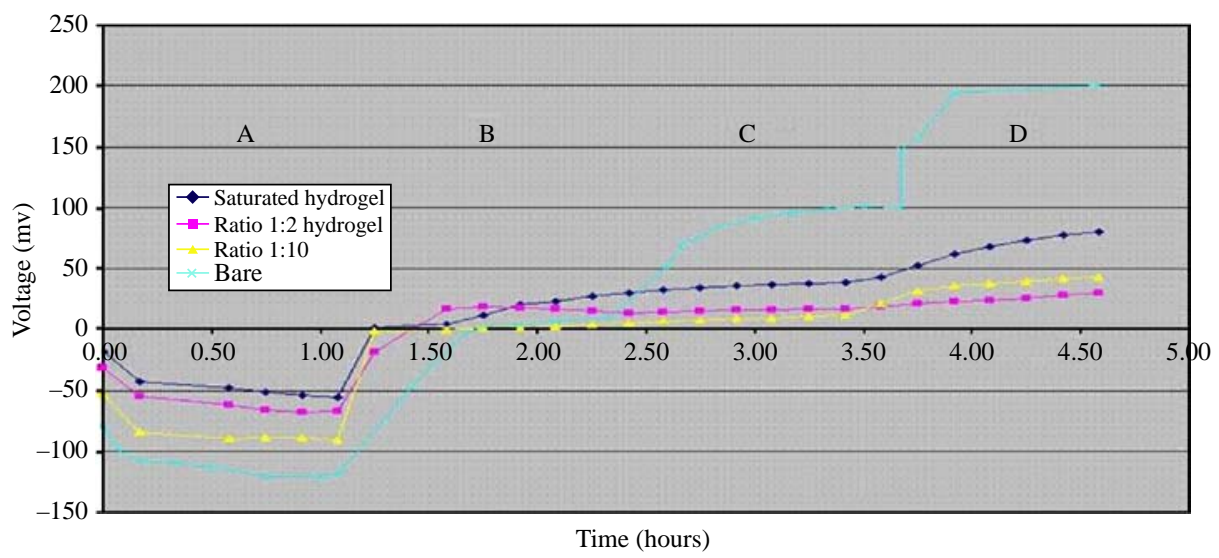

Conversely, the drift rates of the hydrated ESL242SB $/ \mathrm{KCl}$ electrodes were heavily influenced by their initial salt concentration, and this is thought to be a function of the number of "pathways" through which the salt can leach out of the polymer binder. This tends to suggest that higher initial $\mathrm{KCl}$ levels would result in shorter useful lifetimes as a result of higher drift.

The solgel electrodes appear to offer some promise in that they hydrated far more rapidly (within minutes) and hence gave a useful lifetime sooner than their polymer counterparts. One possibility could be to combine these characteristics in a device whereby the polymer electrode surface is covered with a solgel membrane to reduce salt leach out rates and yet present a rapid hydration time.

\section{References}

Andreescu, S., Noguer, T., Magearu, V. and Marty, J-L. (2002), "Screen-printed electrode based on AChE for the detection of pesticides in presence of organic solvents", Talanta, Vol. 57, pp. 169-76.

Atkinson, J.K., Cranny, A.W.J., Glasspool, W.V. and Mihell, J.A. (1999), "An investigation of the performance characteristics and operational lifetimes of multi-element thick film sensor arrays used in the determination of water quality parameters", Sensors and Actuators B: Chemical, Vol. 54 No. 3, pp. 215-31.

Boujtita, M., Hart, J.P. and Pittson, R. (2000), "Development of a disposable ethanol biosensor based on a chemically modified screen-printed electrode coated with alcohol oxidase for the analysis of beer", Biosensors $\mathcal{E}$ Bioelectronics, Vol. 15, pp. 257-63.

Cranny, A.W.J. and Atkinson, J.K. (1998), "Thick film silver-silver chloride reference electrodes", Measurement Science and Technology, Vol. 9 No. 9, pp. 1557-65.

Gac, A., Atkinson, J.K., Zhang, Z. and Sion, R.P. (2002), "A comparison of thick-film chemical sensor characteristics in laboratory and on-line industrial process applications", Measurement Science and Technology, Vol. 13, pp. 2062-73.

Gac, A., Atkinson, J.K., Zhang, Z., Sexton, C.J., Lewis, S.M., Please, C.P. and Sion, R.P. (2004), "Investigation of the fabrication parameters of thick film titanium oxide-PVC $\mathrm{pH}$ electrodes using experimental designs", Microelectronics International, Vols. 21/3, pp. 44-53.

Glasspool, W.V. and Atkinson, J.K. (2003), "An evaluation of the characteristics of membrane materials suitable for the batch fabrication of dissolved oxygen sensors", Microelectronics International, Vol. 20 No. 2, pp. 32-40.

Maminska, R., Dybko, A. and Wroblewski, W. (2006), "All-solid-state miniaturised planar reference electrodes based on ionic liquids", Sensors and Actuators B, Vol. 115, pp. 552-7.

Matsumoto, T., Ohashi, A. and Ito, N. (2002), "Development of a micro-planar $\mathrm{Ag} / \mathrm{AgCl}$ quasi-reference electrode with long-term stability for an amperometric glucose sensor", Analytica Chimica Acta, Vol. 462, pp. 253-9.

Mihell, J.A. and Atkinson, J.K. (1998), "Planar thick-film pH electrodes based on ruthenium dioxide hydrate", Sensors and Actuators B: Chemical, Vol. 48 Nos 1-3, pp. 505-11.

Mroz, A., Borchardt, M., Diekmann, C., Cammann, K., Knoll, M. and Dumschat, C. (1998), "Disposable reference electrode", Analyst, Vol. 123, pp. 1373-6.

Tymeckia, Ł., Zwierkowskab, E. and Koncki, R. (2004), "Screen-printed reference electrodes for potentiometric measurements", Analytica Chimica Acta, Vol. 526, pp. 3-11.

\section{Further reading}

Hassel, A.H., Fushimi, K. and Seo, M. (1999), “An agar-based silver/silver chloride reference electrode for use in microelectrochemistry", Electrochemistry Communications, Vol. 1, pp. 180-3.

Simonis, A., Lüth, H., Wang, J. and Schöning, M.J. (2004), "New concepts of miniaturised reference electrodes in silicon technologyfor potentiometric sensor systems", Sensors and Actuators B, Vol. 103, pp. 429-35.

Tymecki, K., Zwierkowska, E. and Koncki, R. (2004), "Screen-printed reference electrodes for potentiometric measurements”, Anal. Chim. Acta, Vol. 526, pp. 3-11.

\section{Corresponding author}

J.K. Atkinson can be contacted at: jka@soton.ac.uk

To purchase reprints of this article please e-mail: reprints@emeraldinsight.com

Or visit our web site for further details: www.emeraldinsight.com/reprints 